\title{
A DISK INSTABILITY INTERPRETATION FOR THE 2001 OUTBURST OF WZ SGE
}

\author{
Y. Osaki ${ }^{1}$ \\ RFSUMFX
}

Se discutc la explosión en 2001 de WZ SGE basándonos en el modelo de inestabilidad térmica-marea (modelo TTI). S' demuestra que al desarrollo general de la explosión puede ser entendido a través del modelo de inestabilidad del disco y que no se recpuiere transferencia incrementada de masa para explicar las observaciones.

\section{ABSSTRAC'I}

The 2001 ontburst of WZ SGE is discussed basierl on the thermal-tidal instability model (TTI model). It is demonstrated that the overall development of the outburst can be understood by the disk instability model (TTI model) and that no enlanced mass transfer is neederl to explain observations.

\section{Koy Words: ACCRETION, ACCRETION DISKS - BINARIES: CLOSE - STARS: DWARF NOVAE - STARS: INDIVIDUAL (WZ SGE)}

\section{INTTROT)UC'TION}

WZ Sagit.tae. a prototype of one subclass of dwarf novar, called WZ SGE, underwent a full-scale outburst in 2001. July and extensive observations, particularly in optical light, were made for more than one hundred days from the start of eruption to the long fading to cuicsicence with a worldwide collaboration of amateur and professional astronomers (Ishioka et al 2002; Paltcrson et al 2002). I1 is one of the most well-covered observations in the history of dwarf nexa outburst.s. The 2001 outburst of WZ SGE has revealed many intrigning phenomena such as "carly humps", complex superhump light curves. and "echo outbursts". The aim of this paper is to demonstrate that the disk instability model can explain almost all features of its 2001 outburst.

\section{INTERPRETATION OF OBSERVATIONS}

BASED ON THE DISK INSTABILITY NIODEL

\subsection{Recurroner Time}

Two different models hare been proposed to explain the long recurrence time of $11 \%$ SGE of about 30 yrs. One is a model with extremely low viseosity in quiescence (Smak 1993; Osaki 1995; MeverHofmeister of al 1997). The other is a model of a steady or almost stcady cold state with a standard value of viscosity in the cold state but with its inner part truncated (Warner of al 1996: Hameury et al 1997). Wo note that such a disk in the latter model is unable to store the large amount of mass required during the outburst and thus it requires enhanced mass transfer during the outburst.

\footnotetext{
${ }^{1}$ Nagasaki Lniversity. Japan
}

\subsection{Energetics}

Osaki (1995) has already pointed out that the basic outburst energetics of WZ SGE fits to Osaki's (1974) disk instability model in its simplest form: long quiescence of about 30 yrs coupled with a low mass transfer rate $\sim 10^{15} \mathrm{gs}^{-1}$ allow the disk to accumulate mass $\sim 10^{24} \mathrm{~g}$, which is dumped onto the central white dwarf during an outburst. Cannizzo (2001) has shown that the shape of light curve for the 2001 outburst of WZ SGE can be explained by viscous depletion of matter in a disk with an initial mass $\sim 10^{24} \mathrm{~g}$.

\subsection{Early Hump Phenomenon}

Once the thermal instability is started and the disk goes into the hot state, a sudden jump into high viscosity makes the accumulated mass spread inwards and outwards and form a standard hot accretion disk. As discussed by Osaki \& Meyer (2002), the outer edge of the disk, in a binary system with an extremely low mass ratio, not only reaches the $3: 1$ resonance radius but passes it and reaches the far outcr 2:1 resonance radius where the two armed spiral pattern of the Lindblad resonance is excited. The tidal dissipation produces the observed photometric double humps called "arly humps". Osaki \& Meyer (2003) have argued that the $2: 1$ resonance is also responsible for the late appearance of the common superhump in WZ SGE because the 2:1 resonance can suppress the growth of the disk eccentricity due to another resonance called an eccentric "corotation" resonance. 


\subsection{Common Superhumps}

After 12 days of the start of the outburst, the common superhumps finally appeared. Eclipes sudden appeared with the growth of the common superhumps. By synchronous summation of the light curves at the orbital period, Patterson et al (2002) obtained "orbital light curves" that look like those of the quiescent orbital hump. These authors interpreted them as hot spot light curves and eclipses observed as eclipses of the mass transfer hot spot. They then interpreted it as evidence of enhanced "hot spot" and thus enhanced mass transfer rates. However, Osaki \& Meyer (2003) have presented a different interpretation in that superhump light source itself can produce a similar feature in light curves because an arch-like tidal tail is produced by the superhump light dissipation (Murray 1998), which mimics the hot spot light curve.

In this conference, Steeghs (2003) presented Doppler maps of the accretion flow in WZ SGE and he has argued that the sudden brightening of mass transfer stream in the Doppler map with the start of the common superhumps is evidence for enhanced mass transfer. However, I suggest another interpretation for this phenomenon. Since the ballistic mass steam itself does not produce any dissipation, a sudden brightening of the mass transfer stream in emission line Doppler map rather means that the mass transfer steam is either suddenly illuminated by some other light source or an interaction of the mass transfer stream with the disk matter is increased in. the common superhump era. Either one of the two possibilities may occur in the common superhump era. That is, the arch-like tidal tail discussed above can illuminate the mass transfer stream or the interaction of the disk matter with the incoming stream may increase during the common superhump era because of highly time-variable nature of the eccentric precessing disk.

Once the common superhumps emerge, light curves of WZ SGE become extremely complex. The reason why the superhump light curves of WZ SGE are so much complex is that intrinsicaliy timevarying and non-axisymmetric distributions of superhump light sources are coupled with the aspect effects around the binary orbital phases because of its high orbital inclination. Osaki (2003) has proposed a new method of light curve analysis to disentangle these complexities by separating the nonaxisymmetric spatial distribution of the disk from a time variation occurring with the superhump period.
This method is called a "helical tomography" of an accretion disk as a series of disk images is reconstructed by this method. The power spectral data of the superhump light curves by Pat terson et al (2002) are then interpreted under a new concept of helical tomography, and the azimuthal wave numbers of various frequency modes are identified. We hase found a vigorous excitation of a ware component of $\cos (2 \Theta-3 \omega t)$ in the first werk of the common superhump era and this supports Lubow's (1991) theors of non-linear wave coupling of the eerentric Lindblad resonane for the superhump phenomenon.

\subsection{Dips and Echo Outlursts}

The WZ SGE stars often show rebrightening or reflare (called echo outbursts) after the end of the main outburst, the most spectacular "atse wass six consecutive rebrightening of EG ('nc. in 1996/1997. The 2001 outburst of WZ SGE showed the same phenomenon but it had as many as 12 short outbursts with a mean repetition time as short as 2 days. Osaki et al (2001) and Hellior (2001) proposed that ccho outbursts are normal outbursts in the disk where the tidal removal of angular nomentum from the mass reservoir beyond the 3:1 resonance radius feeds matsis into the inner disk and repatrolly excites the thermal instability of the disk.

\section{REFERENCES}

Cannizzo, J. 2001, ApJ, 561, L175

Hameury J.-M., Lasota J.-P., Hure J.-M., 1997, MNR.S. 287,937

Hellier C., 2001, PASP, 113, 78

Ishioka, R., Uemura, M., Kato, 'T.. (', al 2002, Ad:1. 3\$1. L41

Lubow, S.H. 1991, ApJ, 381, 25!9

Meyer-Hofmeister E., Meyer F., Liu B.F., 1998. Ad.A 339,507

Murray, J. R. 1998, MNRAS', 297, 32:3

Osaki Y., 1974, PASJ, 26i. 429

Osaki Y., 1995, PASJ, 47, 47

Osaki, Y., 2003, PAS. , 5.5. 841

Osaki, Y., \& Meyer, F. 2002, A\& A. 38:3. 574

Osaki, Y., \& Meyer, F. 2003, Ad.A, 401, 3:5

Osaki, Y., Meyer, F. \& Meyer-Hofmeister. E. 2001, Al. 370,488

Patterson, J., Masi, G., Richmond. II et al 2002. PASP. 114,721

Smak J., 1993, Acta Astron. 43, 101

Steeghs, D., 2003, in these proceedings

Warner B., Livio M. Tout (…. 1996, MNRAS. 2N2. 735

Yoji Osaki: Faculty of Education, Nagasaki Universiiy, Nagasaki 852-8521, Japan (osaki (inet.nagasaki-u.ac.jp). 